\title{
Exciting vibrons in both frontier orbitals of a single hydrocarbon molecule on graphene
}

\author{
A Mehler ${ }^{1}, \mathbf{N}$ Néel $^{1}$, M-L Bocquet ${ }^{2}$, J Kröger $^{1}$ \\ ${ }^{1}$ Institut für Physik, Technische Universität Ilmenau, D-98693 Ilmenau, Germany \\ ${ }^{2}$ Department of Chemistry, UMR ENS-CNRS-UPMC 8640, Ecole Normale \\ Supérieure, F-75005 Paris, France \\ E-mail: alexander.mehler@tu-ilmenau.de, marie-laure.bocquet@ens.fr
}

\begin{abstract}
Vibronic excitations in molecules are key to the fundamental understanding of the interaction between vibrational and electronic degrees of freedom. In order to probe the genuine vibronic properties of a molecule even after its adsorption on a surface appropriate buffer layers are of paramount importance. Here, vibrational progression in both molecular frontier orbitals is observed with submolecular resolution on a graphene-covered metal surface using scanning tunnelling spectroscopy. Accompanying calculations demonstrate that the vibrational modes that cause the orbital replica in the progression share the same symmetry as the electronic states they couple to. In addition, the vibrational progression is more pronounced for separated molecules than for molecules embedded in molecular assemblies. The entire vibronic spectra of these molecular species are moreover rigidly shifted with respect to each other. This work unravels intramolecular changes in the vibronic and electronic structure owing to the efficient reduction of the molecule-metal hybridization by graphene.
\end{abstract}

Keywords: vibrational progression, single molecules, frontier orbitals, graphene, scanning tunnelling microscopy, quantum chemistry, density functional theory

Submitted to: J. Phys.: Condens. Matter 
Exciting vibrons in both frontier orbitals of a single hydrocarbon molecule on graphene 2

\section{Introduction}

Retaining the appealing genuine characteristics of a free molecule after its anchoring on a surface is highly desirable for fundamental research and technological applications alike. The sharp resonances of a free molecule, for instance, increase the residence time at the molecule for injected electrons and holes, which may then efficiently transfer energy to vibrational degrees of freedom. Such processes enable the analysis of electron-vibration coupling at the single-molecule level. Technologically speaking, the preservation of the free-molecule energy gap between the highest occupied molecular orbital (HOMO) and the lowest unoccupied molecular orbital (LUMO) is relevant to, e.g., light absorption devices conceived for solar energy conversion.

Therefore, it is not surprising that research on appropriate buffer layers that reduce the molecule-metal hybridization has become increasingly important recently. Some seminal achievements in this field may serve as examples. Fluorescence and luminescence of single molecules was recorded on thin films of $\mathrm{Al}_{2} \mathrm{O}_{3}[1,2]$ and $\mathrm{NaCl}$ [2-9]. Spin flip manipulation was reported at the single-atom level on $\mathrm{Al}_{2} \mathrm{O}_{3}$ [10] and $\mathrm{CuN}$ [11] thin films. Vibronic excitations were probed for individual molecules on $\mathrm{Al}_{2} \mathrm{O}_{3}$ [12], $\mathrm{NaCl}$ $[13,14]$ and suitably designed molecular platforms [15-18] while the charging of single atoms [19], the imaging of individual molecular orbitals [20], and chemical reactions at single molecules [21] were performed on bilayers of $\mathrm{NaCl}$.

Two-dimensional materials, e.g., graphene and hexagonal boron nitride (hBN), are emerging as decoupling layers. Predominantly, they have been used as templates to guide the adsorption and intercalation of material [22-35]. Less frequently, single-molecule chemistry has been reported, such as the dehydrogenation of $\mathrm{MH}_{n}$ (M: transition metal) complexes [36,37], non-classical cycloadditions of several phthalocyanine molecules [38], and the controlled abstraction of pyrrolic $\mathrm{H}$ from single phthalcyanines [39] on graphene. While vibrational progression of molecules on ultrathin dielectric films $\left(\mathrm{Al}_{2} \mathrm{O}_{3}, \mathrm{NaCl}, \mathrm{CuN}\right.$, molecular platforms) is ubiquitous [12-18], it has scarcely been reported from two-dimensional materials (graphene, $\mathrm{hBN})$ on semiconductors or metals. Spectroscopy of vibronic levels of 1,3,5-tris $(2,2-$ dicyanovinyl)benzene adsorbed to graphene on a $\mathrm{BN}$-covered $\mathrm{SiO}_{2}$ surface showed the occurrence of pronounced LUMO satellite peaks [40]. Co-Pc molecules deposited on graphene-covered $\mathrm{SiO}_{2} / \mathrm{Si}$ samples were demonstrated to exhibit satellite peaks as well, which were tentatively attributed to excited states or vibrational progression [41]. To date, solely hBN-covered metal surfaces have been used to observe molecular vibronic excitations [42-44]. For instance, the occurrence of sharp molecular resonances in spectra of the differential conductance $(\mathrm{d} I / \mathrm{d} V)$ together with vibrational progression in a single molecular orbital demonstrated the successful reduction of the moleculemetal hybridization by hBN [42]. Open questions in vibrational progression concern the selection of vibrational modes that cause the progression and the electron-vibration coupling in different molecular orbitals.

Here, we use epitaxially grown graphene on $\operatorname{Ir}(111)$ as a buffer layer in order to study 
the vibronic and electronic properties of the hydrocarbon molecule 5,10,15,20-tetraphenylbisbenz $[5,6]$ indeno $\left[1,2,3-c d: 1^{\prime}, 2^{\prime}, 3^{\prime}\right.$-lm $]$ perylene $\left(\mathrm{C}_{64} \mathrm{H}_{36}\right.$, DBP $)$. Low-temperature scanning tunnelling spectroscopy of $\mathrm{d} I / \mathrm{d} V$ unveils vibrational progression in both the HOMO and LUMO of DBP, which enables the estimation of the orbital-dependent electron-vibration coupling. The combination of spectroscopic imaging of individual molecules with the calculated molecular electronic structure unravels the symmetry of the frontier orbitals, which matches the symmetry of vibrations that cause the vibrational progression. Isolated molecules and DBP embedded in a single-layer molecular array exhibit vibronic spectra that are rigidly shifted with respect to each other, which is tentatively attributed to a difference in the local work function.

\section{Experiment and simulations}

The experiments were performed with a low-temperature $(6 \mathrm{~K})$ scanning tunnelling microscope (STM) operated in ultrahigh vacuum $\left(10^{-9} \mathrm{~Pa}\right)$. A clean and singlecrystalline surface was prepared by the bombardment of $\operatorname{Ir}(111)$ with $\mathrm{Ar}^{+}$ions and subsequent annealing. Graphene was epitaxially grown on $\operatorname{Ir}(111)$ by thermal decomposition of $\mathrm{C}_{2} \mathrm{H}_{4}$ (purity: 99.9\%) [45]. DBP molecules were deposited onto graphene-covered $\operatorname{Ir}(111)$ at room temperature by exposing the sample surface to a molecular beam sublimated from a heated Ta crucible. STM images were acquired at constant current with the bias voltage applied to the sample. Constant-height spectra of $\mathrm{d} I / \mathrm{d} V$ were recorded by sinusoidally modulating the bias voltage $\left(5 \mathrm{mV}_{\mathrm{rms}}, 950 \mathrm{~Hz}\right)$ and measuring the current response of the tunnelling junction with a lock-in amplifier. Maps of $\mathrm{d} I / \mathrm{d} V$ were simultaneously recorded with constant-current STM images $\left(20 \mathrm{mV} \mathrm{rms}_{\text {s }}\right.$, $950 \mathrm{~Hz})$.

Ab-initio calculations have been performed in the framework of density functional theory as implemented in the Vienna Ab Initio Simulation Package (VASP) [46]. To model the free DBP molecule, a periodic supercell of $30 \times 20 \times 12 \AA^{3}$ was employed. Ion-electron interaction was described with the Projected Augmented Wave method and the exchange and correlation energy was modeled using the General Gradient Approximation developed by Perdew, Burke and Ernzerhof [47]. The molecules were relaxed until forces were smaller than $0.02 \mathrm{eV} / \AA$. A cut-off energy of $400 \mathrm{eV}$ in the plane-wave expansion was used together with a $1 \times 1 \times 1$ ( $\Gamma$ point) $\mathrm{k}$-grid for the supercell. The smearing technique using a Gaussian broadening of $0.05 \mathrm{eV}$ was applied to achieve electronic convergence. The radical species resulting from the oxidation (cation) and the reduction (anion) of the DBP molecule were optimized. In this case a homogeneous background charge is automatically added to the periodic supercell to ensure its neutrality. As a result, the total energy of a charged solid system includes an additional electrostatic interaction with the charged background that may slightly affect the total energies. The vibrational analysis of the neutral, cationic and anionic forms of DBP was performed by determining vibrational frequencies and modes (eigenvectors), which requires the construction and diagonalization of the Hessian matrix. This 
dynamical matrix was constructed by displacing each atom from its equilibrium position and calculating the forces in each direction. The second derivatives of the total energy are estimated from the forces using linear-response theory as implemented in the 5.4.1 version of VASP. STM images were calculated for the free DBP molecule using isocontours of the electronic density of states $[48,49]$. Isolated molecules lack electronic states of the substrate. In order to compensate for the absence of substrate electronic states, the accuracy of the density functional calculations of the free molecule has been considerably increased by setting the cut-off energy of the plane wave basis to $800 \mathrm{eV}$ (twice the standard value of $400 \mathrm{eV}$ ) and augmenting the number of bands by $25 \%$.

\section{Results and Discussion}

Imaging individual molecules on graphene is an experimentally challenging task even in low-temperature STM experiments due to the often encountered high molecular mobility. In particular lander molecules, such as DBP (figure 1(a)), that are chemically engineered to avoid a strong interaction with the support are subject to tip-induced displacements. Figure 1(b) shows that occasionally the imaging of isolated DBP molecules was feasible. Most likely, imaging was facilitated due to the anchoring of the molecule at a graphene defect site. Indeed, reconstructed point defects in graphene were shown to distort the $\pi$-electron system of graphene giving rise to an increased reactivity at such defects $[50,51]$.

According to the ball-and-stick model of free DBP added to figure 1(b) the four phenyl groups appear with the brightest contrast. The molecular backbone is darker and exhibits uniform contrast, which indicates the parallel adsorption of the molecule to the surface, in agreement with previous reports on metal surfaces [52,53]. Evidence for the otherwise enhanced mobility of DBP on graphene is the dominant occurrence of large molecular islands covering extended regions of graphene (figure 1(c)). A typical close-up view of a molecular island is presented in figure $1(\mathrm{~d})$ where the molecular supercell is spanned by lattice vectors $\mathbf{A}_{1}$ and $\mathbf{A}_{2}$ with $A_{1}=\left|\mathbf{A}_{1}\right|=2.32 \pm 0.05 \mathrm{~nm}$, $A_{2}=\left|\mathbf{A}_{2}\right|=2.01 \pm 0.05 \mathrm{~nm}, \measuredangle\left(\mathbf{A}_{1}, \mathbf{A}_{2}\right)=86 \pm 1^{\circ}$. One of the supercell lattice vectors is aligned with $\langle 11 \overline{2} 0\rangle$ directions of graphene as inferred from STM images resolving the moiré lattice of graphene on $\operatorname{Ir}(111$ ) (inset to figure 1(c)). Further influence of the graphene and moiré lattice on the DBP arrangement is absent since the intermolecular coupling most likely dominates the molecule-substrate interaction at this coverage, as previously reported for $2 \mathrm{H}-\mathrm{Pc}$ on graphene [35]. The unit cell contains two molecules that adopt an arrangement generally referred to as the herringbone assembly, which is driven by the partial compensation of the intrinsic intramolecular quadrupole moment $[53]$.

In a next step $\mathrm{d} I / \mathrm{d} V$ spectra were acquired atop different regions of individual DBP molecules embedded in the herringbone superstructure (figure 2(a)) as well as of isolated DBP (figure 2(b)). Atop a phenyl ring (red spectra in figure 2(a,b)) three peaks labelled $2^{-}-0^{-}$appear at negative bias voltage while $\mathrm{d} I / \mathrm{d} V$ data at positive bias voltage are 
Exciting vibrons in both frontier orbitals of a single hydrocarbon molecule on graphene5

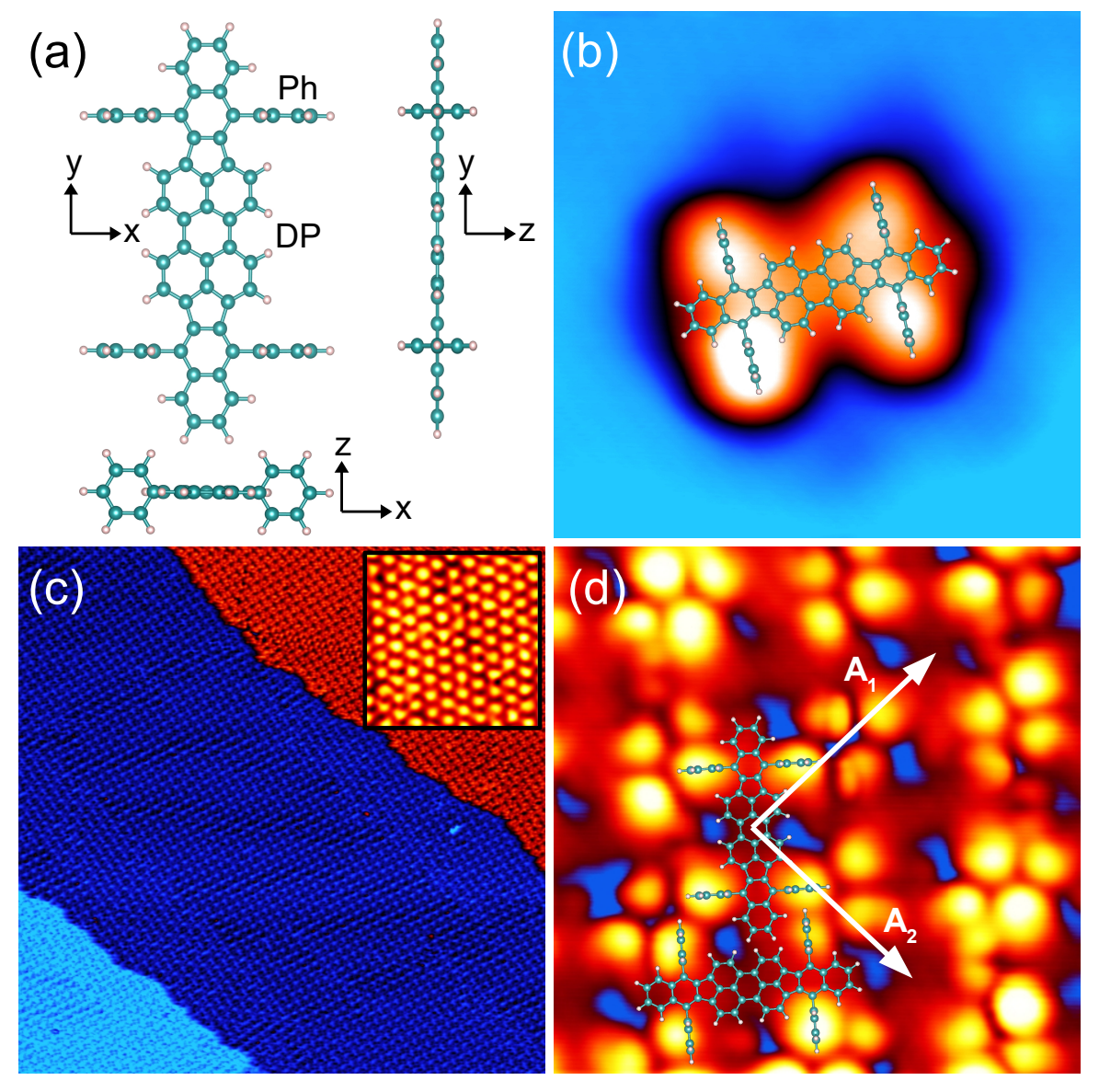

Figure 1. (a) Calculated structure of the free DBP molecule, which exhibits a flat dibenzoperiflanthene (DP) plane with four phenyl $(\mathrm{Ph})$ groups oriented perpendicular to the DP plane. (b) STM image of an isolated DBP molecule on graphene-covered $\operatorname{Ir}(111)$ with superimposed ball-and-stick model $(1 \mathrm{~V}, 50 \mathrm{pA}, 4 \mathrm{~nm} \times 4 \mathrm{~nm})$. The color scale ranges from $0 \mathrm{~nm}$ (light blue) to $0.18 \mathrm{~nm}$ (white). (c) Large-scale STM image showing an island of DBP covering more than three terraces of graphene-covered $\operatorname{Ir}(111)(1 \mathrm{~V}, 50 \mathrm{pA}, 90 \mathrm{~nm} \times 90 \mathrm{~nm}, 0 \mathrm{~nm}$ (light blue) $-0.57 \mathrm{~nm}$ (red)). Inset: STM image of pristine graphene with moiré superstructure $(1.2 \mathrm{~V}, 50 \mathrm{pA}, 25 \mathrm{~nm} \times 25 \mathrm{~nm})$. (d) Close-up view of a DBP island $(1 \mathrm{~V}, 50 \mathrm{pA}, 5 \mathrm{~nm} \times 5 \mathrm{~nm}, 0 \mathrm{~nm}$ (blue) $-0.1 \mathrm{~nm}$ (white)). $\mathbf{A}_{1}, \mathbf{A}_{2}$ span the nearly rectangular unit cell of the molecular superstructure. Ball-andstick models are superimposed to facilitate the identification of individual molecules within the island. The direction of $\mathbf{A}_{1}$ coincides with $\langle 11 \overline{2} 0\rangle$ of graphene.

essentially featureless for both types of molecules. This situation is reversed for spectra recorded at the long edge of the molecular backbone between two phenyl groups (blue spectra). Three (five) peaks labelled $0^{+}-2^{+}\left(0^{+}-4^{+}\right)$are observed at positive bias voltage for DBP embedded in an island (isolated DBP) while at negative polarity the data do not reveal spectral structure. Between $0^{-}$and $0^{+}$a $\mathrm{d} I / \mathrm{d} V$ depletion region with a width of $\approx 3 \mathrm{eV}$ is visible. Remarkably, the spectra for isolated DBP (figure 2(b)) are rigidly shifted with respect to spectra of embedded DBP by $\approx 250 \mathrm{meV}$ towards lower bias voltage. We assign $0^{-}$and $0^{+}$to the spectroscopic signature of the HOMO and 
Exciting vibrons in both frontier orbitals of a single hydrocarbon molecule on graphene6
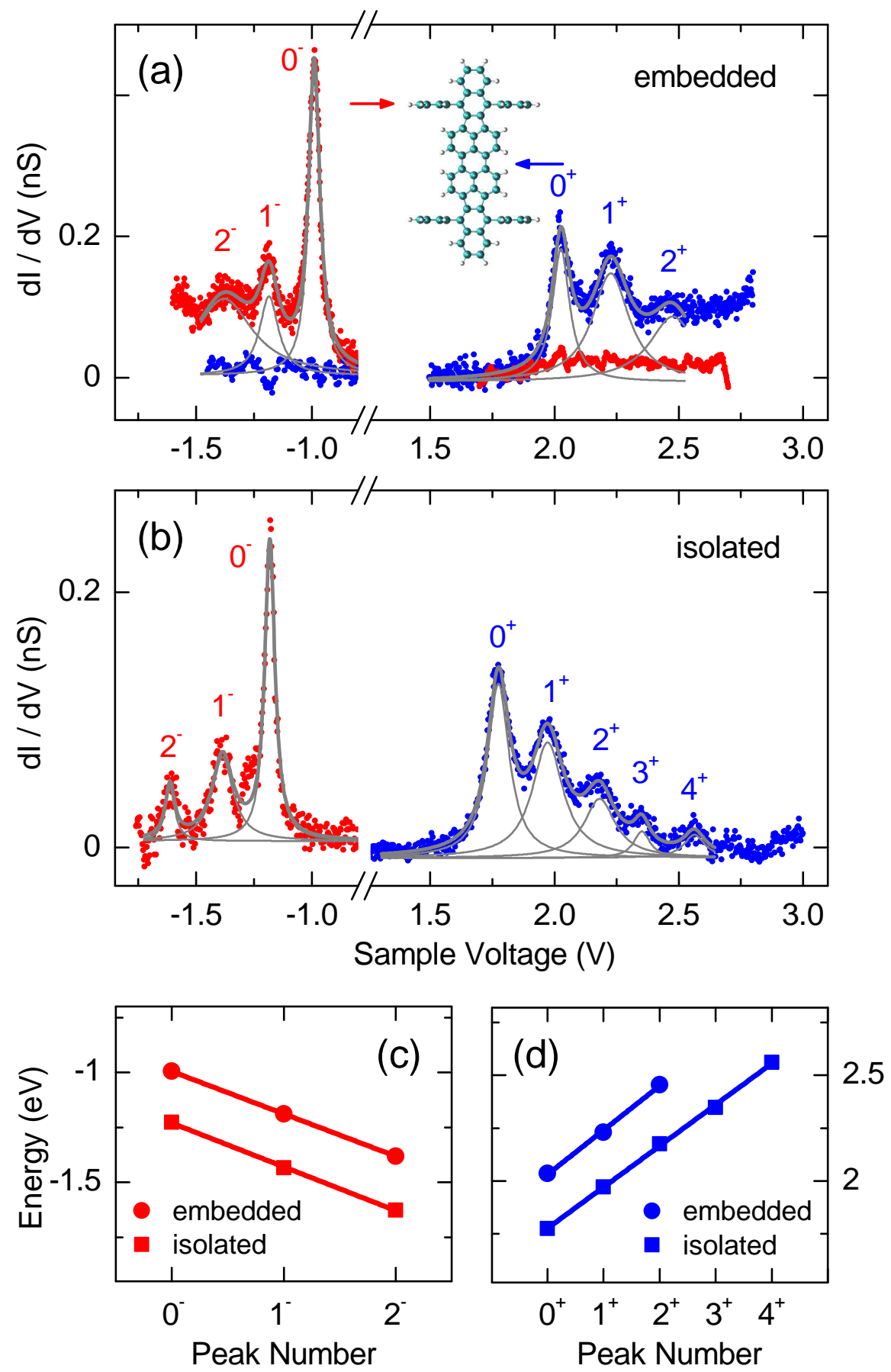

Figure 2. Spatially resolved constant-height $\mathrm{d} I / \mathrm{d} V$ spectra (dots) acquired atop (a) a DBP molecule embedded in an island and (b) an isolated molecule. The positions of spectroscopy are indicated by the arrows in the inset to (a) (red: phenyl ring; blue: long edge of the molecular backbone between two phenyl groups). Solid lines in (a) and (b) represent the superposition (thick) of fits of individual Lorentzian line shapes (thin) to the $\mathrm{d} I / \mathrm{d} V$ data. (c), (d) Energies of peaks $n^{ \pm}(0 \leq n \leq 4)$ visible in the spectra of (a), (b) plotted versus the peak number $n$. Straight lines represent linear fits to the data. 
Exciting vibrons in both frontier orbitals of a single hydrocarbon molecule on graphene 7

Table 1. Energies $(\varepsilon)$ and full widths at half maximum $(\Delta)$ of peaks $n^{ \pm}(0 \leq n \leq 4)$. $\varepsilon$ and $\Delta$ were extracted from fits of Lorentzian line shapes (thin solid lines in figure $2(\mathrm{a}, \mathrm{b}))$ to the spectroscopic data.

\begin{tabular}{cccrl}
\hline & \multicolumn{3}{c}{ embedded } & \multicolumn{2}{c}{ isolated } \\
$n$ & $\varepsilon(\mathrm{eV})$ & $\Delta(\mathrm{eV})$ & $\varepsilon(\mathrm{eV})$ & $\Delta(\mathrm{eV})$ \\
\hline $2^{-}$ & -1.38 & 0.30 & -1.61 & 0.05 \\
$1^{-}$ & -1.19 & 0.10 & -1.37 & 0.11 \\
$0^{-}$ & -0.99 & 0.06 & -1.18 & 0.05 \\
$0^{+}$ & 2.03 & 0.09 & 1.77 & 0.11 \\
$1^{+}$ & 2.23 & 0.18 & 1.97 & 0.15 \\
$2^{+}$ & 2.48 & 0.23 & 2.18 & 0.15 \\
$3^{+}$ & $/$ & $/$ & 2.35 & 0.08 \\
$4^{+}$ & $/$ & $/$ & 2.56 & 0.10 \\
\hline
\end{tabular}

LUMO, respectively. This assignment is corroborated by the comparison of experimental and calculated STM images (supporting information, figure S1).

Plotting the energies of peaks $n^{ \pm}(0 \leq n \leq 4$, table 1) versus the peak sequence (figure $2(\mathrm{c}, \mathrm{d})$ ) unveils a linear variation, which evidences a virtually equidistant separation of the peaks. DBP molecules embedded in islands (circles in figure 2(c,d)) exhibit energy spacings of $209 \pm 8 \mathrm{meV}$ for $0^{+}-2^{+}$and $193 \pm 3 \mathrm{meV}$ for $2^{-}-0^{-}$. The average energy separation between adjacent peaks for isolated DBP (squares) is determined as $199 \pm 4 \mathrm{meV}$ for $0^{+}-4^{+}$and $195 \pm 4 \mathrm{meV}$ for $2^{-}-0^{-}$. These findings are reminiscent of vibrational progression where a tunnelling electron or hole is injected into the discrete vibronic states of the transiently charged molecule yielding replica of the orbital signature in $\mathrm{d} I / \mathrm{d} V$ spectra. An important prerequisite for vibrational progression to occur is the presence of sharp molecular resonances and, thus, the extended lifetime of the transient charge state of the molecule [54], which is fulfilled here owing to the weak DBP-metal hybridization [39,55]. The peaks $n^{ \pm}(n \geq 1)$ are not due to other molecular orbitals since calculations of the free molecule revealed that the HOMO-1 (LUMO+1) is $1 \mathrm{eV}$ below (above) the HOMO (LUMO) energy [53].

The spatial regions within an individual molecule where vibrational progression occurs can readily be determined by spectroscopic imaging at bias voltages corresponding to the peaks $n^{ \pm}(n \geq 1)$. Figure 3 shows maps of $\mathrm{d} I / \mathrm{d} V$ recorded above a DBP molecule embedded in an island at $-1.2 \mathrm{~V}\left(1^{-}\right),-1 \mathrm{~V}\left(0^{-}\right), 2 \mathrm{~V}\left(0^{+}\right), 2.2 \mathrm{~V}\left(1^{+}\right)$. All $\mathrm{d} I / \mathrm{d} V$ data were normalized in order to minimize the influence of the varying tip-surface distance in constant-current imaging [56]. As expected for vibrational progression [57] the $\mathrm{d} I / \mathrm{d} V$ maps are similar for $1^{-}, 0^{-}\left(0^{+}, 1^{+}\right)$. At negative bias voltage $\left(1^{-}, 0^{-}\right)$strong $\mathrm{d} I / \mathrm{d} V$ signal is mainly localized at the DBP phenyl groups, while at positive bias voltage $\left(0^{+}, 1^{+}\right)$the edges of the DBP backbone exhibit enhanced contrast. The presence of adjacent molecules in the densely packed herringbone superstructure leads to slight deviations from symmetric patterns. According to the assignment of $0^{-}\left(0^{+}\right)$to the HOMO (LUMO) the regions of high contrast in $\mathrm{d} I / \mathrm{d} V$ maps of $1^{-}$, 
Exciting vibrons in both frontier orbitals of a single hydrocarbon molecule on graphene8
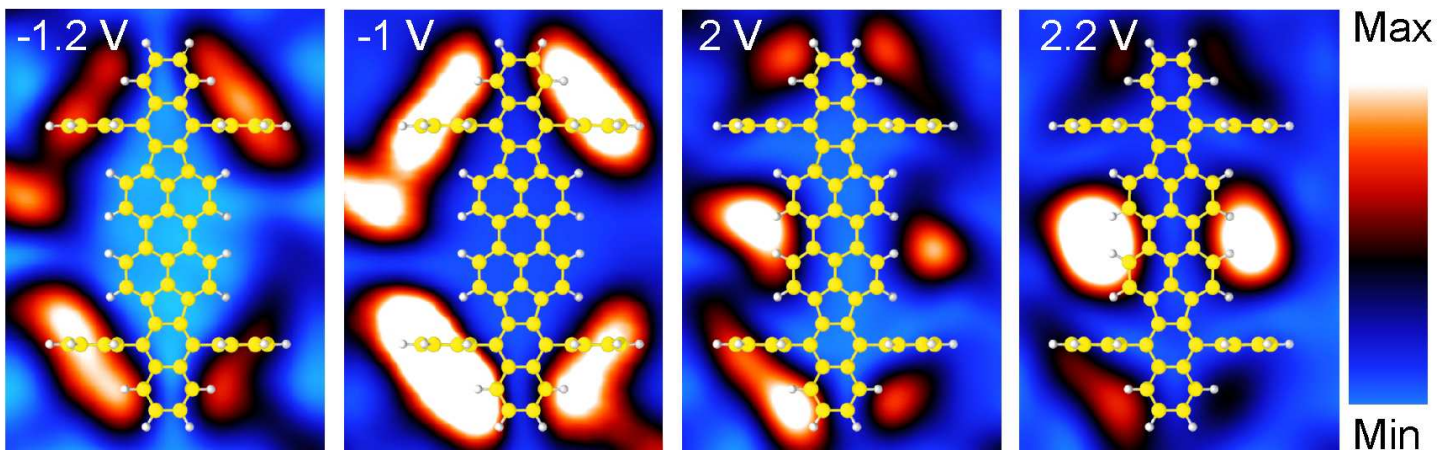

Figure 3. Normalized constant-current $\mathrm{d} I / \mathrm{d} V$ maps of a single DBP molecule embedded in an island recorded at $-1.2 \mathrm{~V}\left(1^{-}\right),-1 \mathrm{~V}\left(0^{-}\right), 2 \mathrm{~V}\left(0^{+}\right), 2.2 \mathrm{~V}\left(1^{+}\right)$and $50 \mathrm{pA}(1.70 \mathrm{~nm} \times 2.35 \mathrm{~nm})$. Ball-and-stick models are superimposed in order to identify the position of the molecule.

$0^{-}\left(0^{+}, 1^{+}\right)$represent the spatial distribution of the HOMO (LUMO) density of states (DOS). Moreover, the spatial variation of the DOS provides a natural explanation for the observed different behavior of $\mathrm{d} I / \mathrm{d} V$ spectra at negative and positive bias voltage (figure 2(a,b)). The spectroscopic signal is strong (weak) at intramolecular sites where the corresponding molecular orbital is present (absent).

An interesting question concerns the actual vibrational modes participating in the vibrational progression. In a previous work for molecules adsorbed to graphene on BN-covered $\mathrm{SiO}_{2}[40]$ the energy-dependent electron-vibration coupling was calculated, which peaked in a narrow energy range and, thus, enabled the identification of vibrational quanta relevant to the progression. Here, we follow a different approach and compare the symmetries of electronic and vibrational excitations. To this end, the neutral, cationic and anionic forms of the free DBP molecule was calculated on the basis of density functional theory. Assuming the vacuum character of DBP is reasonable due to its efficient decoupling from the metal surface via graphene, in agreement with previous work for other molecules adsorbed on graphene $[30,39,55,58$ 60]. Electron energy loss spectra (supporting information, figure S2) reveal energy losses that virtually coincide with vibration energies calculated for the free molecule [52], which corroborates the free-molecule character of DBP on graphene. Our calculations for free DBP yield 12 (8) in-plane deformation modes of the molecular backbone (of the phenyl groups in the normal plane) in an energy range of $194.1 \mathrm{meV} \leq \hbar \omega \leq 199.3 \mathrm{meV}$ $(192.2 \mathrm{meV} \leq \hbar \omega \leq 194.8 \mathrm{meV})$ ( $\hbar \omega$ : vibrational energy with $\hbar$ the Planck constant, supporting information, table S1). Since the energies of these modes differ by less than $5 \mathrm{meV}$ the broad $(\geq 50 \mathrm{meV}$, table 1 ) replica peaks of the HOMO and LUMO hamper the unambiguous assignment of vibrational progression to a single mode. As the calculations show (vide infra), the coupling of the inelastic tunnelling electron to several modes occurs [61], in agreement with observations reported for Sn-Pc molecules adsorbed to a molecular buffer layer on $\operatorname{Ag}(111)$ [18].

The optimized structures of the neutral (figure 1(a)), cationic and anionic forms 
(a)
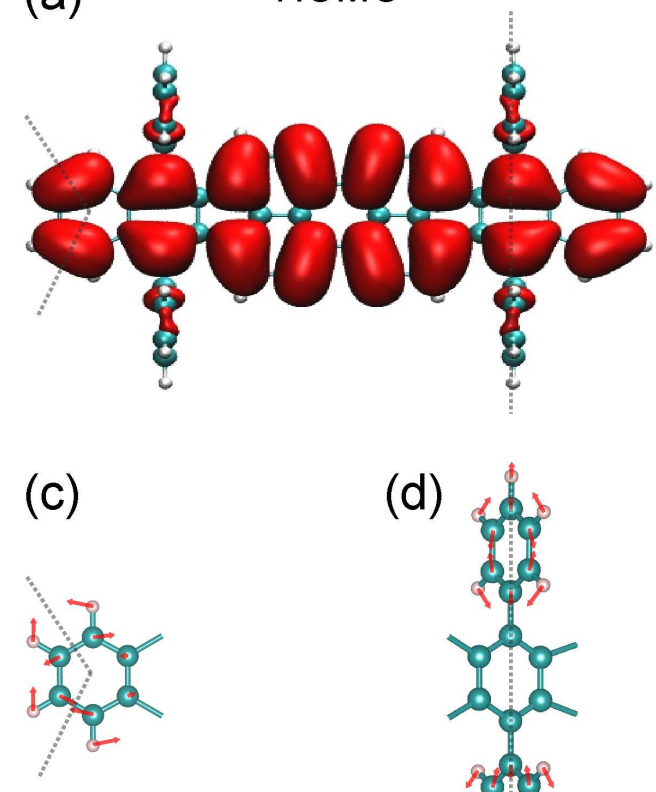

(b)

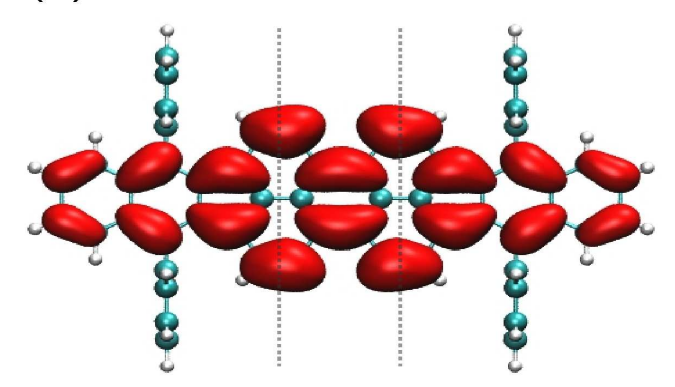

(e)

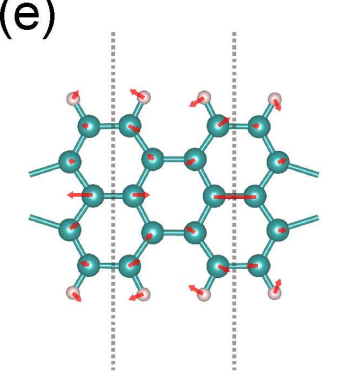

Figure 4. Density functional calculations of the DBP frontier orbitals and vibrational analysis. Spatial density of the HOMO (a) and the LUMO (b) frontier orbitals of neutral DBP. Gray dotted lines depict symmetry planes of the local orbital structure. (c)-(e) Close-up views of molecular regions indicated by the mirror planes in (a), (b). The displacements of $\mathrm{H}$ (white) and $\mathrm{C}$ (gray) marked by arrows display $\mathrm{C}-\mathrm{C}$ stretch and $\mathrm{C}-\mathrm{H}$ bending modes at the DP plane edge (c), in the two Ph planes (d), and in the center of the DP plane (e). The calculated energies of the vibrational quanta are $199.0 \pm 0.1 \mathrm{meV}, 198.2 \pm 0.1 \mathrm{meV}, 197.4 \pm 0.2 \mathrm{meV}$ for (c), (d), (e), respectively.

of the free molecule are very similar. All calculated structures display a flat dibenzoperiflanthene (DP) plane with four phenyl $(\mathrm{Ph})$ groups oriented perpendicular to this plane. The calculated HOMO (figure 4(a)) and LUMO (figure 4(b)) of neutral DBP exhibit different characteristics and symmetries. Notably, the HOMO shows substantial electron density on $\mathrm{Ph}$, while it is negligible for the LUMO. The HOMO has a central nodal plane indicating the absence of electron density at the center of the molecule, while the LUMO presents antinodes of the electron density in this plane. In figure $4(\mathrm{a}, \mathrm{b})$ local symmetry planes of the electron density distribution are indicated by gray dotted lines at the edge of the DP plane, at the $\mathrm{Ph}$ groups, and at the central part of the DP plane. These symmetry elements of the electronic states match the symmetry of three vibrational normal modes that are illustrated in figure $4(\mathrm{c}, \mathrm{d}, \mathrm{e})$. These modes are energetically degenerate around $200 \mathrm{meV}$ and equivalent within a few meV range for the neutral, cationic and anionic forms of DBP. Therefore, molecular deformations upon charge attachment as predicted by the (pseudo) Jahn-Teller effect [62] do not play a role in our study. Moreover, this energy corresponds well to the equidistant 
Exciting vibrons in both frontier orbitals of a single hydrocarbon molecule on graphene 10

Table 2. Electron-vibration coupling parameters extracted from the ratio of $1^{ \pm}$and $0^{ \pm}$for embedded and isolated DBP molecules.

\begin{tabular}{lll}
\hline & $1^{-} / 0^{-}$ & $1^{+} / 0^{+}$ \\
\hline embedded & $0.26 \pm 0.05$ & $0.82 \pm 0.09$ \\
isolated & $0.28 \pm 0.05$ & $0.74 \pm 0.07$ \\
\hline
\end{tabular}

spacings between the orbital replica observed in the vibrational progression (figure 2). Therefore, the matching symmetry of electronic and vibrational states is an important ingredient for the selection of vibrational modes that cause the vibrational progression. For example, electrons extracted from the HOMO at negative bias voltage couple to two degenerate vibrations, namely the in-plane and out-of-plane $\mathrm{C}-\mathrm{H}$ bending modes of the DP edge (figure 4(c)) and the $\mathrm{Ph}$ groups (figure 4(d)). Similarly, electrons injected into the LUMO at positive bias voltage couple to in-plane $\mathrm{C}-\mathrm{H}$ bending modes of the DP edge (figure 4(c)) and to $\mathrm{C}-\mathrm{C}$ stretch modes in the $\mathrm{DP}$ central region (figure $4(\mathrm{e}))$. The regions of these active modes appear with bright contrast in maps of $\mathrm{d} I / \mathrm{d} V$ (figure 3). Many more DBP vibrational quanta (20 in total) appear in the energy range 190-200 meV. Some of these quanta exhibit atom displacements that share the same symmetry as the normal modes depicted in figure $4(\mathrm{c}, \mathrm{d}, \mathrm{e})$. Other quanta represent a mixture of the highly symmetric modes. The presented vibrational analysis enables the detailed understanding of the vibronic STS spectra and highlights the key ingredient for vibrational progression, namely the compatible symmetries of vibrational and electronic states. A similar conclusion has recently been drawn from angle-resolved photoelectron spectroscopy experiments [63].

Next, differences in the spectroscopic signatures of vibrational progression for embedded and isolated molecules will be addressed as well as the orbital-dependent electron-vibration coupling strengths and the rigid shift of the entire vibronic spectra.

First, comparing the vibrational progression for DBP in a molecular island (figure 2(a)) and isolated DBP (figure 2(b)) reveals that the vibronic features are more clearly resolved for the isolated molecule. In particular, the LUMO of isolated DBP exhibits more replica than the LUMO of DBP embedded in the island. While a clear-cut explanation to this observation is currently not available, the presence of neighbouring molecules in the molecular islands is likely the reason for the broadened replica in the following sense. The periodic arrangement of DBP in the herringbone superstructure may convert discrete vibrational levels of the isolated molecule into dispersing bands, which would contribute to a broadening and the suppression of higher-order replica peaks in the vibronic spectra. In addition, it was previously shown that the close proximity of molecules may induce intermolecular coupled vibrations with energies between the fundamental vibrational modes of the single molecule [64].

Second, the dimensionless electron-vibration coupling strengths were extracted. In a previous resonant-tunneling model [65-67] describing the electron transport across a double-barrier junction via a single electronic level coupled to a phonon bath the 
coupling parameter was defined as $\lambda=M^{2} /(\hbar \omega)^{2}$ ( $M$ : matrix element of the coupling between electron and vibration). By calculating the total transmission of the junction it was further demonstrated that $\lambda$ may approximately be extracted from the height ratio of the first satellite $\left(1^{ \pm}\right)$and main $\left(0^{ \pm}\right)$peak $[65,66]$. Table 2 summarizes these values for the embedded and isolated molecule. The obtained coupling strengths are comparable with previously reported values for vibrational progression in a single orbital of Co-Pc molecules on hBN [42]. Since in the experiments reported here vibrational progression occurs in both frontier orbitals, investigations into orbital-dependent electron-vibration coupling are possible. While the presence of adjacent molecules does not strongly alter the coupling strength (columns in table 2), the electron-vibration coupling constant does vary for the different frontier orbitals (rows). Electrons injected into the LUMO couple more strongly to the specified DBP vibrational quanta than holes injected into the HOMO. The enhanced coupling of charge injected into molecular orbitals to vibrational degrees of freedom is in agreement with previous reports on local heating $[68,69]$ and two-level conductance fluctuations [70]. However, in the present case this observation may be surprising since the LUMO resonance width of $0.10 \mathrm{eV}$ indicates a shorter residence time for the injected electron than for the hole attached to the HOMO with a width of $0.06 \mathrm{eV}$ (table 1 ). Therefore, the number of vibrational quanta involved in the progression may play an additional role in the following sense. The DP plane hosts more vibrational modes (35 out of 61) with energies that are compatible with the vibrational progression than the Ph groups (supporting information, table S1). Consequently, vibrational progression in the LUMO, which is located at the long sides of the DP plane (figure 3 and supporting information, figure S1), reflects the coupling to a higher number of vibrational modes than the $\mathrm{HOMO}$ at the $\mathrm{Ph}$ groups (figure 3 and supporting information, figure S1) leading to a higher signal strength of the replica peaks.

Third, the entire vibronic spectrum of the isolated DBP molecule is rigidly shifted to lower bias voltage by $\approx 250 \mathrm{mV}$ compared to the spectrum of DBP embedded in the herringbone superstructure. At first sight an obvious reason for the shift may be the absence of a molecular environment for the isolated molecule. Indeed, reducing the number of polar $\mathrm{H}-\mathrm{O}$ bonds was reported to shift the LUMO and LUMO+1 of PTCDA on $\mathrm{Au}(111), \mathrm{Au}(788)$ by $\approx 350 \mathrm{meV}$ to lower bias voltage [71]. However, polar $\mathrm{H}-\mathrm{O}$ bonds are absent in the case of DBP molecules. Moreover, different molecular assemblies were reported for $\mathrm{DBP}$ on $\mathrm{Au}(111)$, which did not entail variations in $\mathrm{d} I / \mathrm{d} V$ spectra [72]. Therefore, the molecular environment plays a minor role in the rigid shift of the vibronic spectra with respect to each other. A shift of the molecular resonances due to a variation of the local electric field between tip and sample, as reported for noble-metal surface states $[73,74]$, can be excluded because of the nearly invariant tip height above embedded and isolated molecules. Rather, we propose a change in the local work function that drives the shift in the present case. The weak DBP-metal coupling gives rise to the lateral independence of the DBP electron affinity and ionization potential, which define, respectively, the LUMO and HOMO energy with respect to the 
surface vacuum level [75]. As a result, local variations of the latter would entail a corresponding shift of LUMO and HOMO energies, as reported for the adsorption of porphyrine molecules at different sites of $\mathrm{hBN}$ on $\mathrm{Cu}(111)$ [76]. In the present case the local change of the work function is assigned to the presence of a defect site of the graphene lattice, at which the isolated DBP molecule has most likely been trapped. Indeed, Stone-Wales defects that occur during the epitaxial growth of graphene were demonstrated to lower the work function of defect-free graphene on the order of $100 \mathrm{mV}$ $[77,78]$.

\section{Conclusion}

The observation of vibrational progression in both frontier orbitals of a molecule on graphene combined with density functional calculations of the molecular electronic and vibrational structure has unraveled several aspects of vibronic excitations at the singlemolecule level. Replica of molecular orbitals in vibrational progression are induced by vibrations whose atom displacement patterns match the symmetry of the electronic states they couple to. The number of replica depends on the atomic environment; that is, isolated molecules exhibit a more pronounced progression than molecules hybridized within a molecular assembly. The hybridization reduces the number and increases the width of replica peaks. The strength of replica resonances reflects the orbitaldependent electron-vibration coupling and the number of vibrational quanta involved in the progression. Local variation of the work function can lead to the rigid shift of the entire vibronic spectrum.

\section{Acknowledgments}

The authors thank E Runge (Ilmenau), G Rouillé, T Fritz (Jena) and G Schull (Strasbourg) for discussions. Financial support by the Deutsche Forschungsgemeinschaft through Grant No.KR 2912/12-1 are acknowledged. M-L B thanks the French computational center GENCI (Grant 2017-project: A0010807364) for computer ressources.

\section{References}

[1] Qiu X H, Nazin G V and Ho W 2003 Science 299 542-546

[2] Chen C, Chu P, Bobisch C A, Mills D L and Ho W 2010 Phys. Rev. Lett. 105(21) 217402

[3] Ćavar E, Blüm M C, Pivetta M, Patthey F, Chergui M and Schneider W D 2005 Phys. Rev. Lett. 95(19) 196102

[4] Dong Z C, Zhang X L, Gao H Y, Luo Y, Zhang C, Chen L G, Zhang R, Tao X, Zhang Y, Yang J L and Hou J G 2009 Nature Photonics 4 50-54

[5] Zhang Y, Luo Y, Zhang Y, Yu Y J, Kuang Y M, Zhang L, Meng Q S, Luo Y, Yang J L, Dong Z C and Hou J G 2016 Nature 531 623-627

[6] Doppagne B, Chong M C, Lorchat E, Berciaud S, Romeo M, Bulou H, Boeglin A, Scheurer F and Schull G 2017 Phys. Rev. Lett. 118(12) 127401 
Exciting vibrons in both frontier orbitals of a single hydrocarbon molecule on graphene 13

[7] Zhang Y, Meng Q S, Zhang L, Luo Y, Yu Y J, Yang B, Zhang Y, Esteban R, Aizpurua J, Luo Y, Yang J L, Dong Z C and Hou J G 2017 Nature Communications 815225

[8] Imada H, Miwa K, Imai-Imada M, Kawahara S, Kimura K and Kim Y 2017 Phys. Rev. Lett. 119(1) 013901

[9] Kröger J, Doppagne B, Scheurer F and Schull G 2018 Nano Letters 18 3407-3413

[10] Heinrich A J, Gupta J A, Lutz C P and Eigler D M 2004 Science 306 466-469

[11] Hirjibehedin C F, Lutz C P and Heinrich A J 2006 Science 312 1021-1024

[12] Qiu X H, Nazin G V and Ho W 2004 Phys. Rev. Lett. 92(20) 206102

[13] Wang Y, Kröger J, Berndt R and Tang H 2010 Journal of the American Chemical Society 132 $12546-12547$

[14] Pavliček N, Swart I, Niedenführ J, Meyer G and Repp J 2013 Phys. Rev. Lett. 110(13) 136101

[15] Frederiksen T, Franke K J, Arnau A, Schulze G, Pascual J I and Lorente N 2008 Phys. Rev. B 78(23) 233401

[16] Matino F, Schull G, Köhler F, Gabutti S, Mayor M and Berndt R 2011 Proceedings of the National Academy of Sciences 108 961-964

[17] Hauptmann N, Hamann C, Tang H and Berndt R 2013 The Journal of Physical Chemistry C 117 9734-9738

[18] Schwarz F, Wang Y F, Hofer W A, Berndt R, Runge E and Kröger J 2015 The Journal of Physical Chemistry C 119 15716-15722

[19] Repp J, Meyer G, Olsson F E and Persson M 2004 Science 305 493-495

[20] Repp J, Meyer G, Stojković S M, Gourdon A and Joachim C 2005 Phys. Rev. Lett. 94(2) 026803

[21] Liljeroth P, Repp J and Meyer G 2007 Science 317 1203-1206

[22] Berner S, Corso M, Widmer R, Groening O, Laskowski R, Blaha P, Schwarz K, Goriachko A, Over H, Gsell S, Schreck M, Sachdev H, Greber T and Osterwalder J 2007 Angewandte Chemie International Edition 46 5115-5119

[23] Goriachko A, He, Knapp M, Over H, Corso M, Brugger T, Berner S, Osterwalder J and Greber T 2007 Langmuir 23 2928-2931

[24] N'Diaye A T, Gerber T, Busse C, Mysliveček J, Coraux J and Michely T 2009 New Journal of Physics 11103045

[25] Pan Y, Gao M, Huang L, Liu F and Gao H J 2009 Applied Physics Letters 95093106

[26] Mao J, Zhang H, Jiang Y, Pan Y, Gao M, Xiao W and Gao H J 2009 Journal of the American Chemical Society 131 14136-14137

[27] Sicot M, Bouvron S, Zander O, Rüdiger U, Dedkov Y S and Fonin M 2010 Applied Physics Letters 96093115

[28] Pollard A, Perkins E, Smith N, Saywell A, Goretzki G, Phillips A, Argent S, Sachdev H, Müller F, Hüfner S, Gsell S, Fischer M, Schreck M, Osterwalder J, Greber T, Berner S, Champness N and Beton P 2010 Angewandte Chemie International Edition 49 1794-1799

[29] Sutter E, Albrecht P, Wang B, Bocquet M L, Wu L, Zhu Y and Sutter P 2011 Surface Science $6051676-1684$

[30] Roos M, Künzel D, Uhl B, Huang H H, Brandao Alves O, Hoster H E, Gross A and Behm R J 2011 Journal of the American Chemical Society 133 9208-9211

[31] Cavallin A, Pozzo M, Africh C, Baraldi A, Vesselli E, Dri C, Comelli G, Larciprete R, Lacovig P, Lizzit S and Alfè D 2012 ACS Nano 6 3034-3043

[32] Järvinen P, Hämäläinen S K, Banerjee K, Häkkinen P, Ijäs M, Harju A and Liljeroth P 2013 Nano Letters 13 3199-3204

[33] Halle J, Néel N and Kröger J 2016 The Journal of Physical Chemistry C 120 5067-5073

[34] Banerjee K, Kumar A, Canova F F, Kezilebieke S, Foster A S and Liljeroth P 2016 The Journal of Physical Chemistry C $\mathbf{1 2 0} 8772-8780$

[35] Néel N and Kröger J 2017 Molecules 22731

[36] Donati F, Dubout Q, Autès G, Patthey F, Calleja F, Gambardella P, Yazyev O V and Brune H 2013 Phys. Rev. Lett. 111(23) 236801 
Exciting vibrons in both frontier orbitals of a single hydrocarbon molecule on graphene 14

[37] Altenburg S J and Berndt R 2014 New Journal of Physics 16093047

[38] Altenburg S J, Lattelais M, Wang B, Bocquet M L and Berndt R 2015 Journal of the American Chemical Society 137 9452-9458

[39] Néel N, Lattelais M, Bocquet M L and Kröger J 2016 ACS Nano 10 2010-2016

[40] Riss A, Wickenburg S, Tan L Z, Tsai H Z, Kim Y, Lu J, Bradley A J, Ugeda M M, Meaker K L, Watanabe K, Taniguchi T, Zettl A, Fischer F R, Louie S G and Crommie M F 2014 ACS Nano 8 5395-5401

[41] Bouvron S, Maurand R, Graf A, Erler P, Gragnaniello L, Skripnik M, Wiedmann D, Engesser C, Nef C, Fu W, Schönenberger C, Pauly F and Fonin M 2018 Nanoscale 10(3) 1487-1493

[42] Schulz F, Drost R, Hämäläinen S K and Liljeroth P 2013 ACS Nano 7 11121-11128

[43] Palma C A, Joshi S, Hoh T, Ecija D, Barth J V and Auwärter W 2015 Nano Letters 15 2242-2248

[44] Liu L, Dienel T, Widmer R and Gröning O 2015 ACS Nano 9 10125-10132

[45] Hattab H, N'Diaye A T, Wall D, Jnawali G, Coraux J, Busse C, van Gastel R, Poelsema B, Michely T, zu Heringdorf F J M and von Hoegen M H 2011 Applied Physics Letters 98141903

[46] Kresse G and Furthmüller J 1996 Comput. Mater. Sci. 6 15-50

[47] Perdew J P, Burke K and Ernzerhof M 1996 Phys. Rev. Lett. 77(18) 3865-3868

[48] Tersoff J and Hamann D R 1983 Phys. Rev. Lett. 50 1998-2001

[49] Bocquet M L, Lesnard H, Monturet S and Lorente N 2009 Theory of Elastic and Inelastic Electron Tunneling (Weinheim, Germany: Wiley-VCH) 9783527625482

[50] Boukhvalov D W and Katsnelson M I 2008 Nano Letters 8 4373-4379

[51] Cretu O, Krasheninnikov A V, Rodríguez-Manzo J A, Sun L, Nieminen R M and Banhart F 2010 Phys. Rev. Lett. 105(19) 196102

[52] Rouillé G, Kirchhuebel T, Rink M, Gruenewald M, Kröger J, Forker R and Fritz T 2015 Phys. Chem. Chem. Phys. 17(45) 30404-30416

[53] Kirchhuebel T, Gruenewald M, Sojka F, Kera S, Bussolotti F, Ueba T, Ueno N, Rouillé G, Forker R and Fritz T 2016 Langmuir 32 1981-1987

[54] Franke K J and Pascual J I 2012 J. Phys.: Condens. Matter 24394002

[55] Endlich M, Gozdzik S, Néel N, da Rosa A L, Frauenheim T, Wehling T O and Kröger J 2014 The Journal of Chemical Physics 141184308

[56] Ziegler M, Néel N, Sperl A, Kröger J and Berndt R 2009 Phys. Rev. B 80(12) 125402

[57] Huan Q, Jiang Y, Zhang Y Y, Ham U and Ho W 2011 The Journal of Chemical Physics 135 014705

[58] Dou W, Huang S, Zhang R Q and Lee C S 2011 The Journal of Chemical Physics 134094705

[59] Bazarnik M, Brede J, Decker R and Wiesendanger R 2013 ACS Nano 7 11341-11349

[60] Järvinen P, Hämäläinen S K, Ijäs M, Harju A and Liljeroth P 2014 The Journal of Physical Chemistry C 118 13320-13325

[61] Coropceanu V, Cornil J, da Silva Filho D A, Olivier Y, Silbey R and Brédas J L 2007 Chemical Reviews 107 926-952

[62] Bersuker I B 2017 Journal of Physics: Conference Series 833012001

[63] Graus M, Grimm M, Metzger C, Dauth M, Tusche C, Kirschner J, Kümmel S, Schöll A and Reinert F 2016 Phys. Rev. Lett. 116(14) 147601

[64] Han Z, Czap G, Xu C, Chiang C l, Yuan D, Wu R and Ho W 2017 Phys. Rev. Lett. 118(3) 036801

[65] Wingreen N S, Jacobsen K W and Wilkins J W 1988 Phys. Rev. Lett. 61(12) 1396-1399

[66] Wingreen N S, Jacobsen K W and Wilkins J W 1989 Phys. Rev. B 40(17) 11834-11850

[67] Gadzuk J W 1991 Phys. Rev. B 44(24) 13466-13477

[68] Pecchia A, Romano G and Di Carlo A 2007 Phys. Rev. B 75(3) 035401

[69] Schulze G, Franke K J, Gagliardi A, Romano G, Lin C S, Rosa A L, Niehaus T A, Frauenheim T, Di Carlo A, Pecchia A and Pascual J I 2008 Phys. Rev. Lett. 100(13) 136801

[70] Néel N, Kröger J and Berndt R 2011 Nano Letters 11 3593-3596

[71] Kröger J, Jensen H, Berndt R, Rurali R and Lorente N 2007 Chem. Phys. Lett. $438249-253$

[72] Mehler A, Kirchhuebel T, Néel N, Sojka F, Forker R, Fritz T and Kröger J 2017 Langmuir 33 
Exciting vibrons in both frontier orbitals of a single hydrocarbon molecule on graphene 15

$6978-6984$

[73] Limot L, Maroutian T, Johansson P and Berndt R 2003 Phys. Rev. Lett. 91(19) 196801

[74] Kröger J, Limot L, Jensen H, Berndt R and Johansson P 2004 Phys. Rev. B 70(3) 033401

[75] Ishii H, Sugiyama K, Ito E and Seki K 1999 Advanced Materials 11 605-625

[76] Joshi S, Bischoff F, Koitz R, Ecija D, Seufert K, Seitsonen A P, Hutter J, Diller K, Urgel J I, Sachdev H, Barth J V and Auwärter W 2014 ACS Nano 8 430-442

[77] Jee H g, Jin K H, Han J H, Hwang H N, Jhi S H, Kim Y D and Hwang C C 2011 Phys. Rev. B 84(7) 075457

[78] Bae G, Cha J, Lee H, Park W and Park N 2012 Carbon 50851 - 856 